\title{
Current Status and Prospects of Supercomputing Used for Gas Turbine Engines Design
}

\author{
Omair Alhatim \\ National Center for Jet Engine Technology, King Abdul-Aziz City for Science and Technology, Riyadh 11442, Saudi Arabia
}

Received: December 21, 2015 / Accepted: January 06, 2016 / Published: February 29, 2016.

\begin{abstract}
The engineering analysis techniques used for the GTE (gas turbine engines) design are presented, the physical effects, which impact is not currently taken into account are described, further research directions to strengthen core design competencies are identified, the requirements for computing power are formulated. Internal cooling techniques for gas turbine blades have been studied for several decades. The internal cooling techniques of the gas turbine blade includes: jet impingement, rib turbulated cooling, and pin-fin cooling which have been developed to maintain the metal temperature of turbine vane and blades within acceptable limits in this harsh environment.
\end{abstract}

Key words: Engineering analysis, GTE design, aerodynamics of impeller machines, heat-and-mass transfer, blade internal cooling.

\section{Introduction}

The engineering calculation is the primary mean to reduce the cost of new products designing in mechanical engineering, because it avoids long-term preliminary development phase [1]. Therefore, virtual engineering, which includes the research and optimization of geometrical and physical properties of the product, is the most progressive current trend in the evaluation environment [2]. Using computer simulations in a virtual environment is a mandatory component of the core competence of the company, working in high-tech engineering market, such as gas turbine engines. Core competencies, by definition [3], are the complex interaction of specific individual technologies and skills, that should provide potential access to different markets, determine the specific advantages of the company's final product and should be virtually non-reproducible by competitors. The core competence of turbine engine manufacturing is the design of the gas generator (high-pressure loop). The fact of possession of this competence determined by

Corresponding author: Omair Alhatim, Dr., research assistant professor, research field: jet propulsion. three factors:

- design of modern high efficiency heavy loaded impeller machines;

- design of turbine cooled blades;

- design of effective combustion chambers with low hazardous emission.

\section{Aerodynamics of Impeller Machines}

The dominant current aerodynamic modelling approach for design of compressors and GTE (gas turbine engines) turbines is the solution of steady and unsteady RANS (Reynolds (Favre) averaged Navier-Stokes) equations. The main concept of this method involves substituting of the instantaneous values of a turbulent flow with the sum of their time-averaged values, and the deviations from the average values. This simplifies the Navier-Stokes equations, but results in the appearance of additional variables which determining requires the introduction of new equations. For example, in $k-\varepsilon$ model, two additional equations for transport of the turbulent kinetic energy and transport of turbulence dissipation are solved. There are also other commonly used differential turbulence models: $k-\omega$, The SST (shear 
stress transport), Spalart and Allmaras, etc. Their main advantages are the relative simplicity and low costs of the calculations. To ensure the reliability of the obtained results such models include experimental model constants. In most cases, the RANS approach provides a satisfactory accuracy at design computation stage using typical personal computers. Since the grid size for the spatial steady aerodynamics computation of a single blade passage of the compressor or uncooled turbine blade is approximately 300,000 units (using the "wall-adjacent" functions in the boundary layer), which requires a 400-450 Mb RAM (random-access memory) unstructured for CFD (computational fluid dynamics) - unstructured solver.

However, the above approach does not provide a high degree reliable characterization of such physical effects as a unsteady "stator-rotor" blading interaction of turbo-machines (stator-the stationary blading, rotor-the rotating blading), the flow trajectory behind blades and detached shocks, detailed formation on the boundary layer at the blade surface, its interaction with the radial gap flow, separating and eddy currents, their effect on the overall aerodynamic efficiency of the compressor or the turbine [4]. These physical effects, being the parameters of uncertainties during design, force the designer to over-design the airflow for up to $5 \%$ and the COP (coefficient of performance) for up to $3 \%$. Currently, the compressor stall margin in the entire operating range also cannot be reliably predicted. Finally, considering the units build errors in, the task of meeting of all the design technical specification requirements becomes too difficult. Fig. 1 shows a typical flow structure in the supersonic turbo-machine stage (compressor stage).

Fig. 2 shows the vortex flow in the inter-blade channel of the turbomachine (turbine).

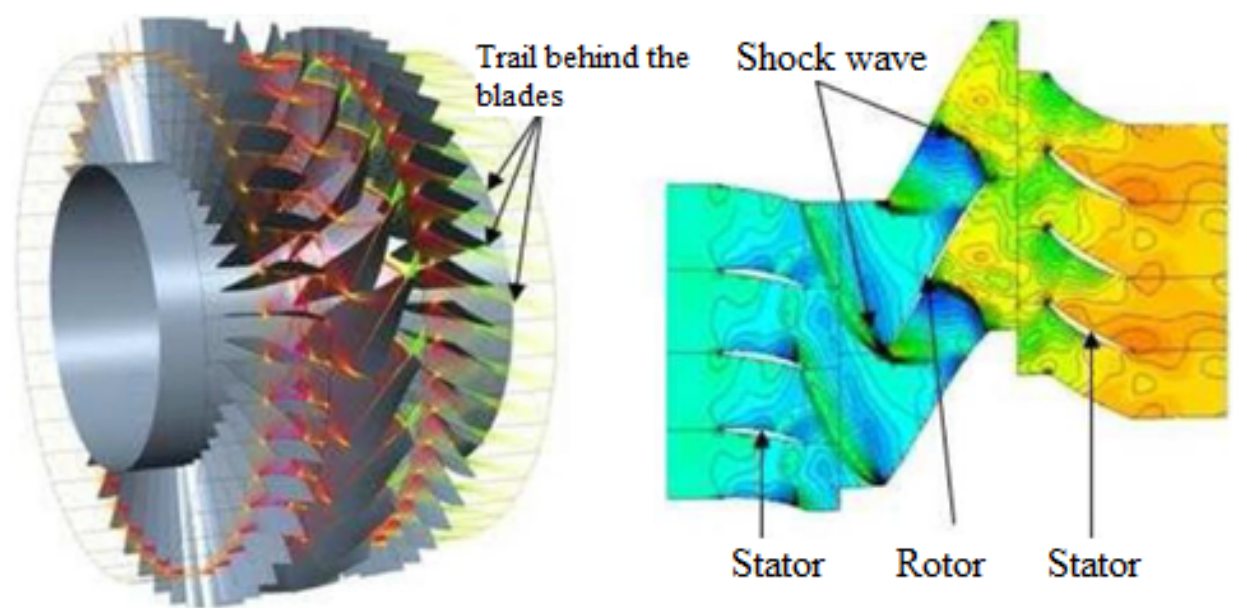

Fig. 1 Turbo-machine stage (stator-rotor-stator).

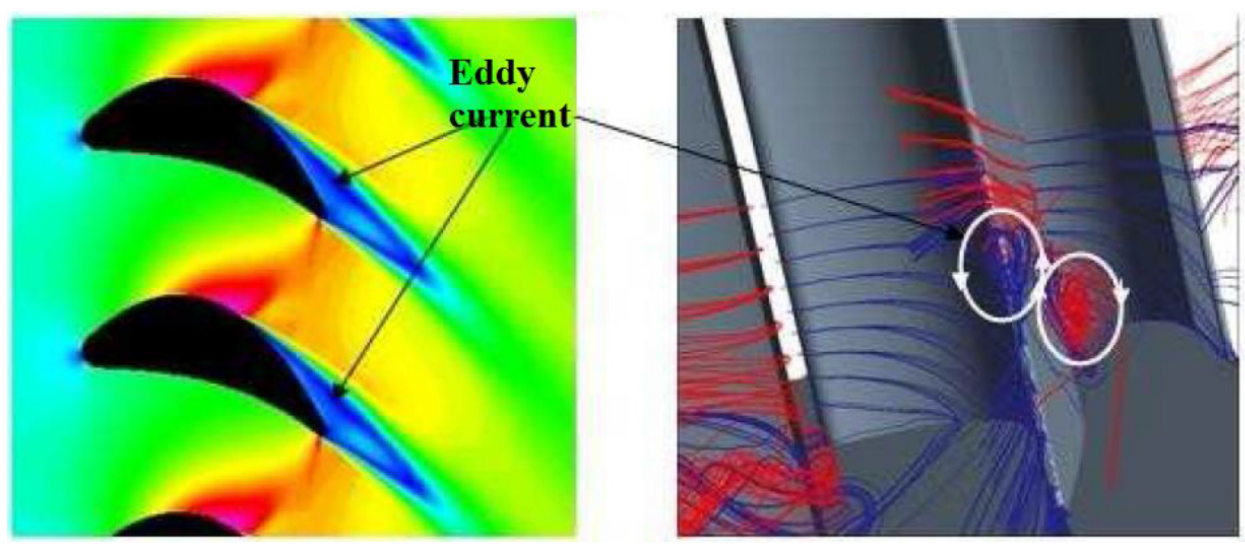

Fig. 2 Blade channel of the turbine row. 


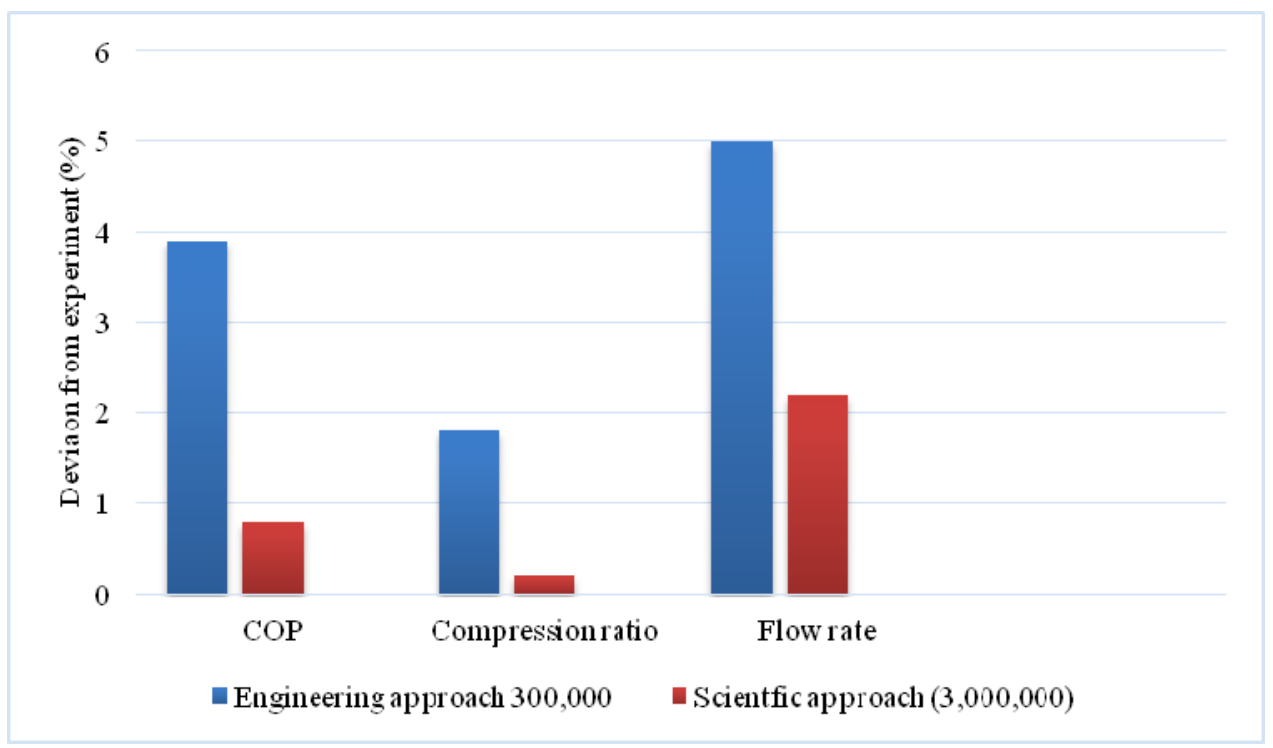

Fig. 3 The compressor stage aerodynamic modelling uncertainty for different approaches.

The solution of the problem of ensuring a reliable turbo-machine aerodynamics is possible under the following conditions:

- experimental study of fundamental physical process in turbo-machine wheelspace (boundary layers and eddy currents, shock waves, flow trajectory behind blades, "stator-rotor" blading interaction);

- development of mathematical models and numerical simulation methods for aerodynamics based on the experiment.

Up to date advanced scientific approaches, such as LES (large eddy simulation ) and the DES (detached eddy simulation), as well as direct numerical simulation, require one or even two orders of magnitude more detailed computation grids and appropriate computing resources that are not available today for engineering applications. Fig. 3 shows the modelling uncertainty of the aerodynamic characteristics of the turbo-machine stage when RANS-traditional approach (grid size of 3,000,000 units) is used and advanced LES-modelling (grid size of 3,000,000 units).

Examples of the use of these models to solve practical problems of national engine-building are rare today and, first and foremost, deal with solving the unsteady aerodynamics and acoustics of the fan and the nozzle of the gas turbine engine. However, the growth trends of processors productivity and computing power of supercomputers suggest that such approaches will be increasingly applied to solve practical problems in upcoming years.

\section{Heat-and-Mass Transfer}

The main objective of heat and mass transfer in a gas turbine engine is to determine thermal condition of the most loaded elements. One of the most complex elements of the engine is turbine-cooled blade. Thermal condition of the blade is determined by solving of the thermal conduction differential equation. The solving of this equation is not difficult. The main difficulty is the definition of the second type boundary conditions (the temperature of the medium, which contacts the metal and the heat transfer coefficient between the medium and the metal of the blade). Therefore, the solution of the thermal condition determination problem is a part of the solution of the external hot gas flow over the blade section and internal coolant flow inside the blade. Conventionally, the heat transfer coefficient between the metal of the blade and the medium is determined based on semi-empirical experimental correlations that provide an acceptable level of forecast precision at the design stage. The limited usage range 


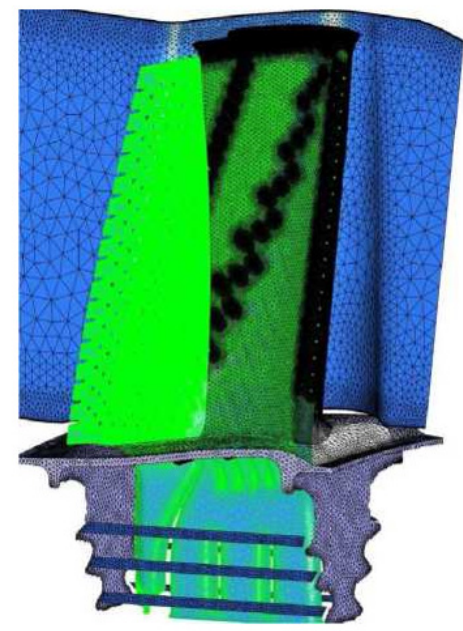

(a)

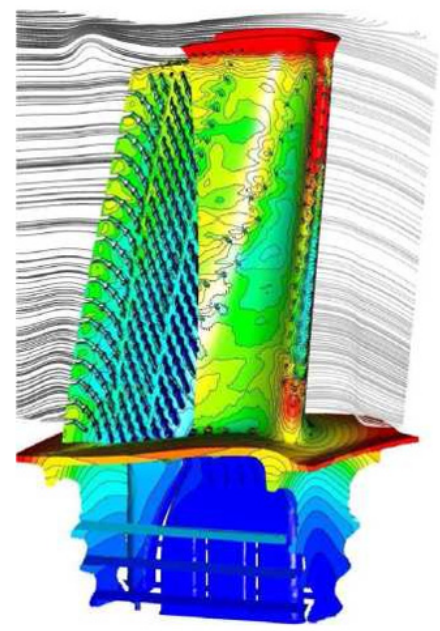

(b)

Fig. 4 Numerical simulation of conjugate heat condition of turbine cooled blade: (a) computational grid of inner cavity of the blade, (b) temperature pattern of the blade.

of criteria correlations, especially for modern heavy loaded turbine blades is a disadvantage of this method. At the stage of spatial optimization of turbine blade. the forecast precision of the thermal condition based on generalized experimental data is insufficient due to inability to describe the spatial effects of the interaction of the boundary layers with each other and with the shock wave, effects of the boundary layer transition from laminar to turbulent state, and so the relaminarization effects, etc. [5].

The most promising engineering approach to the definition of heat and mass transfer in a turbine-cooled blade is a solution of the conjugate formulation problem, when the heat balance of "hot gas-metal-cooling air" system is determined based on joint iterative solution of averaged Navier-Stokes equations and the energy equation. This approach avoids the determination of the heat transfer coefficient, which is an uncertainty factor in determination of the metal blade temperature. This can account virtually all significant factors, determining the heat transfer. The main disadvantage of this approach is the high requirements for the grid discretisation and computing resources. Considering the complexity of the geometry of the object, the grid size for determination of the thermal condition of the cooled blades with a loop or cyclone convection-film cooling system is not less than 6 million units, which requires at least $8 \mathrm{~GB}$ of RAM. Fig. 4 shows an example of a grid for turbine cooled rotor blade and the result of calculation of the conjugate heat transfer on it.

Solving a similar problem in an unsteady random access memory, RAM-formulation already requires at least 12 GB of RAM, and application of parallelizing at least to 80 processor cores to achieve an acceptable computation time. Solving the problem using, for example, MT8-method requires an order of magnitude increase of the grid size and parallelizing to 600-800 processor cores. At the same time, to ensure appropriate forecast precision, the identification of mathematical models based on physical experiment (heat transfer in intensive cyclone formation, laminar-turbulent transition, and relaminarization) is required.

\section{Combustion Processes}

There are several basic requirements that applied to modern combustion chambers: ensuring a high level of combustion efficiency, the rated value of the circumferential and radial distortion of the temperature pattern at the inlet of the turbine and the low hazardous emission into the atmosphere. Design of the 
combustion chamber is one of the greatest challenges in creating a gas turbine engine. The main reason for this is the complexity of the fuel combustion process. First, the combustion process is affected by the quality of fuel and oxidant (air) mixture. During atomization and mixing of the liquid fuel, an interaction of fuel droplets with each other and with an oxidant, evaporation of fuel droplets, their dispersion and coagulation are taking place. This has resulted in the air-fuel mixture formation, which mixing quality depends on the design features of swirlers and atomizers in combustion chamber. Most of the design and preliminary development of the combustion chamber today performed using a full-scale experiment. Methods of numerical simulation based on KAIB-approach allow at this time to determine the aerodynamics (losses) in the combustion chamber with satisfactory quality. Fig. 5a presents fragments of a grid

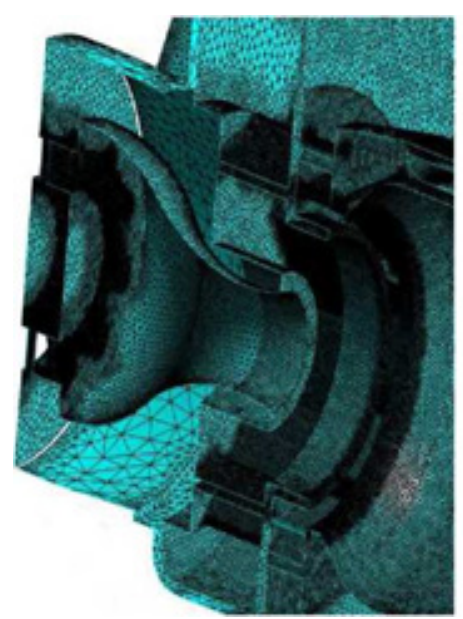

(a) model and Fig. $5 b$-the velocity pattern in the cross section, located near the front wall.

During numerical simulation of the combustion process, the prediction error of temperature pattern beyond the combustion chamber in average is up to $5 \%$ (in local areas of up to 20\%) and the hazardous emission-up to $300 \%$. During numerical simulation, using the engineering approach, the atomization and mixing characteristics are taken into account indirectly and chemical kinetics of the combustion process is based on the consideration of 2-3 main reactions. However, used combustion models are semi-empirical (contain the coefficients of the model, chosen based on model experiments), and have a limited range of applications. Grid size of the numerical simulation of the combustion chamber sector with atomizers and swirlers in RANS-formulation is not less than 15 million units. Fig. 6 shows the temperature field in the

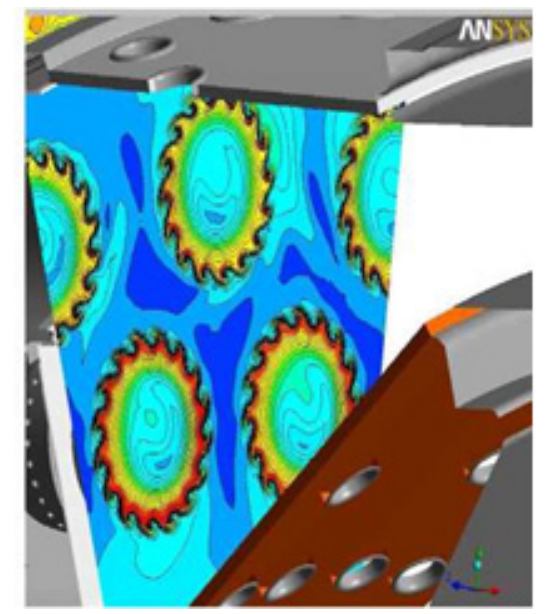

(b)

Fig. 5 Numerical simulation of aerodynamics of combustion chamber: (a) fragment of a computation grid, (b) velocity pattern.

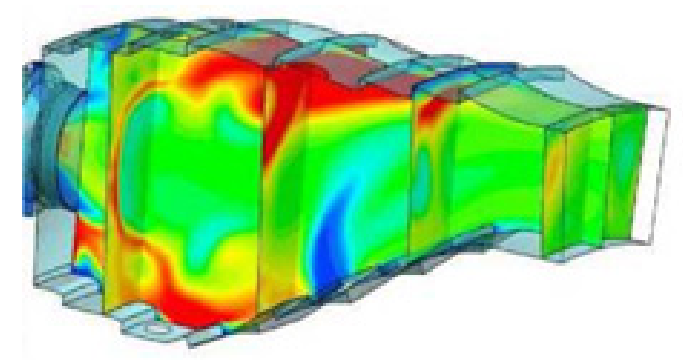

(a)

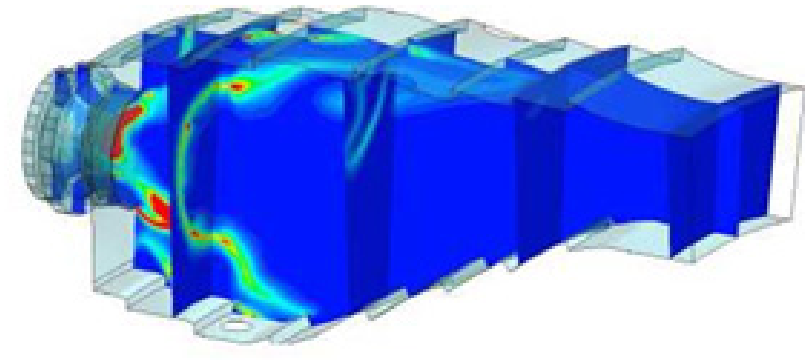

(b)

Fig. 6 Numerical simulation of the combustion process in combustion chamber: (a) temperature pattern in the combustion chamber, (b) NOx reaction rate. 
CC (combustion chamber) of GTE and the rate of hazardous substances formation.

The use of more advanced methods such as DES to LES for evaluation of processes in the combustion chamber, requires five times larger grid size in comparison with RANS-approach and parallelization to 1,000 cores to obtain an acceptable computation time. Numerical simulation of the full-scale combustion chamber increases these requirements for another order.

\section{Internal Cooling of Turbine Blade}

The internal cooling of the gas turbine blade is influenced by the channel aspect ratio, turbulator configurations, rotational and flow parameters. The cooling channels are either single-pass (with radial outward flow) or multi-pass (both radial outward and radial inward flow).

Coolant is circulated through serpentine passages fabricated on the inside of the gas turbine blade in order to remove heat from the blade surface. Different aspect ratio channels are applicable to different parts of the turbine blade as shown in Fig. 7.

A typical cooled turbine blade is shown in Fig. 8 (courtesy of Han, et al. [6]). As shown, the blade is hollow, so cooling air can pass through it internally. A number of traditional cooling concepts are provided in the hollow internal passage of turbine blades which are suitable in different portion of the blades.

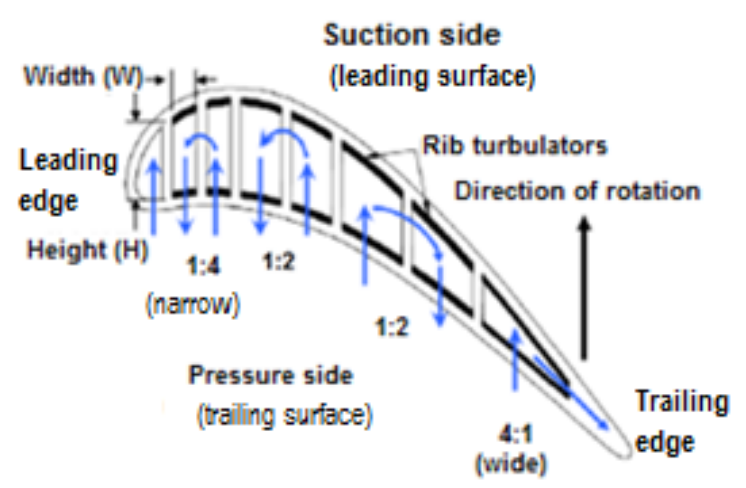

Fig. 7 Gas turbine blade internal cooling channels and their applicable aspect ratios.

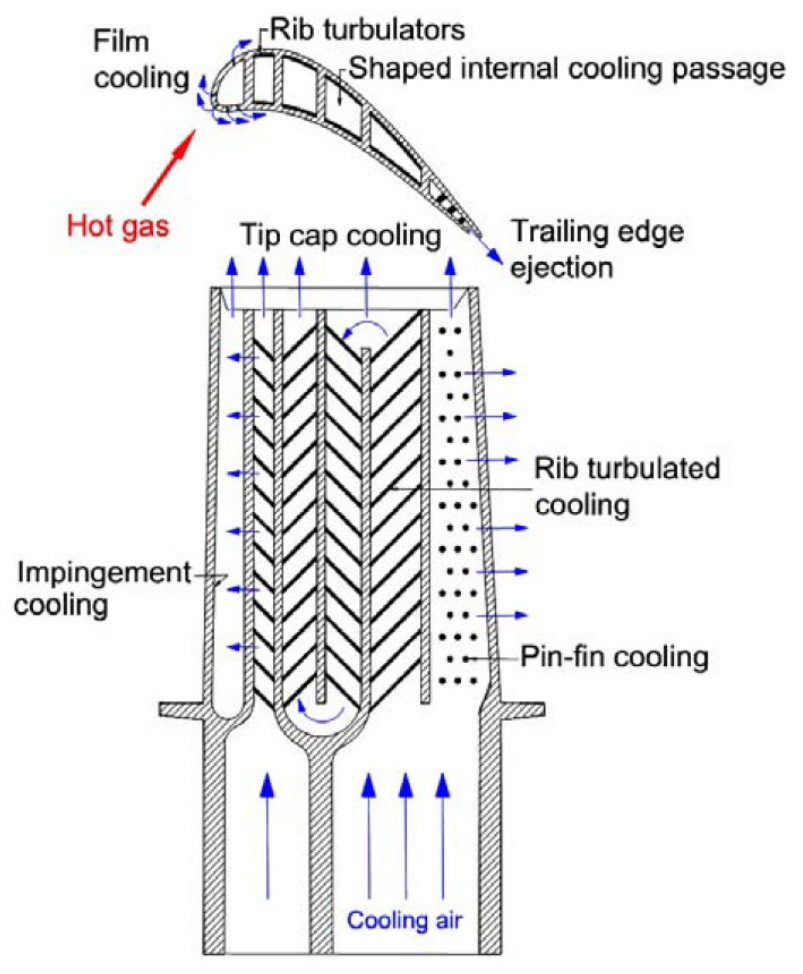

Fig. 8 Typical blade cooling.

\subsection{Impingement Cooling}

Impingement cooling is commonly used near the leading edge of the airfoils, where the heat loads are the greatest. With the cooling jets striking (impinging) the blade wall, the leading edge is well suited for impingement cooling because of the relatively thick blade wall in this area. Impingement can also be used near the mid-chord of the blades. The effect of jet-hole size and distribution, cooling channel crosssection, and target surface shape all have significant effects on the heat transfer coefficient distribution. It has been shown by Metzger, et al. [7] that, multiple jets perform very differently from a single jet striking a target surface. They concluded that, for multiple jets, the Nusselt number is strongly dependent on the Reynolds number. florschuetz and $\mathrm{Su}$ [8] reported that, cross-flow decreases the overall heat transfer from the impingement surface. Because the enhancement from the impingement jets is much greater than the convective enhancement, the overall Nusselt numbers decrease in cross-flow. 


\subsection{Pin-Fin Cooling}

Due to manufacturing constraints in the very narrow trailing edge of the blade, pin-fin cooling is typically used to enhance the heat transfer in this region. The pins typically have a height-to-diameter ratio between 0.5 and 4. In a pin-fin array, heat is transferred from both the smooth channel endwall and the numerous pins. Flow around the pins in the array is comparable to flow around a single cylinder. As the coolant flows past the pin, the flow separates and wakes are shed downstream of the pin. In addition to this wake formation, a horseshoe vortex forms just upstream of the base of the pin, and the vortex wraps around the pins. This horseshoe vortex creates additional mixing, and thus enhanced heat transfer. Many factors must be considered when investigating pin-fin cooling. The type of pin-fin array and the spacing of the pins in the array, the pin size and shape have a profound impact on the heat transfer in the cooling passage. Because pin-fins are commonly coupled with trailing edge ejection, the effect of this coolant extraction must also be considered.

\subsection{Pin Array and Partial Length Pin Arrangement}

There are two array structures commonly used. One is the inline array and the other is the staggered array. Fig. 9 shows a typical experimental test model with a staggered array of pin-fins. Metzger, et al. [9] used staggered arrays of circular pins with 1.5-5 pin

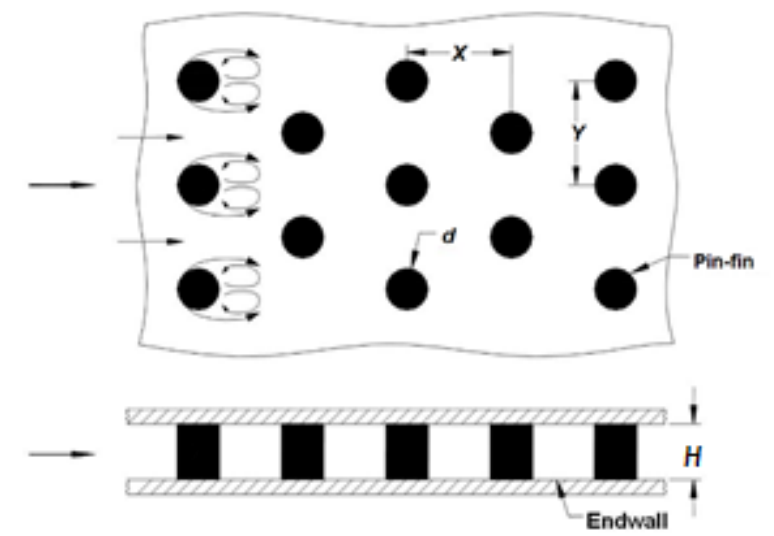

Fig. 9 A typical test model and secondary flow for pin-fin cooling studies. diameter spacing in a rectangular channel. A closer spaced array (smaller $x / D$ ) shows a higher heat transfer coefficient. Chyu, et al. [10] showed that, the heat transfer coefficient on the pin surface for both arrays is consistently higher than that of the channel endwall. The pin surface heat transfer is observed to be $10 \%-20 \%$ higher for the presented case.

\subsection{Effect of Pin Shape and Array Orientation}

Metzger, et al. [11] reported the effect of flow incident angle on oblong pins. All incident angles except $90^{\circ}$ yield higher Nusselt numbers than circular pins. The $\gamma=90^{\circ}$ array yields significantly lower Nusselt numbers. The $\gamma= \pm 30^{\circ}$ array has the highest Nusselt numbers, about $20 \%$ higher than the circular pin array on the average. Except for $\gamma=90^{\circ}$, the pressure drop for oblong pins are significantly higher than circular pins because of flow turning due to oblong pins. Chyu [12] studied the effect of a fillet at the base of the cylindrical pin. It is interesting to note that the fillet cylinder inline formation has better heat transfer than the straight cylinders in the inline formation. Chyu, et al. [13] used cube and diamond shaped pins and reported that, cube-shaped pins have the highest mass transfer coefficients among the shapes considered and round pins have the lowest mass transfer coefficients. Corresponding pressure loss coefficients are higher for the cube and diamond shaped pins relative to the circular pins.

\section{Requirements to Computing Resources}

Improving accuracy of prediction of aerodynamic processes, heat and mass transfer and combustion in gas turbine engines can be achieved by:

- Experimental study of the factors affecting the state of the boundary layer in the turbo-machine wheelspace, processes of atomization and mixing of the fuel;

- Develop reduced models of chemical kinetics, describing the process of fuel combustion;

- Development of mathematical models on the basis on the experimental studies; 
Table 1 Computing power required for problem solving of engineering analysis during GTE design by LES/DES eddy simulation methods.

\begin{tabular}{|c|c|c|c|c|c|c|}
\hline \multirow{2}{*}{ Problem } & \multicolumn{2}{|c|}{ Grid size (million cells) } & \multicolumn{2}{|c|}{ Required memory (Gb) } & \multicolumn{2}{|c|}{ Number of processor cores } \\
\hline & Steady & Unsteady & Steady & Unsteady & Steady & Unsteady \\
\hline $\begin{array}{l}\text { Aerodynamics of turbo-machine uncooled } \\
\text { stage }\left(\text { sector } 10-12^{\circ}\right)\end{array}$ & 5 & 9 & 7 & 13 & 35 & 63 \\
\hline $\begin{array}{l}\text { Aerodynamics of turbo-machine cooled } \\
\text { stage }\left(\text { sector } 10-12^{\circ}\right)\end{array}$ & 30 & 54 & 42 & 75 & 210 & 380 \\
\hline Conjugate heat and mass transfer & 50 & 90 & 70 & 125 & 350 & 630 \\
\hline $\begin{array}{l}\text { Aerodynamics and combustion in } \\
\text { combustion chamber (atomizer, sector } 20^{\circ} \text { ) }\end{array}$ & 75 & 135 & 105 & 190 & 525 & 950 \\
\hline
\end{tabular}

- Improving of problem-solving techniques based on mentioned models, ensured parallelism (thousands and tens of thousands of processor cores) on computers with distributed memory.

The above requirements for computing resources, meeting of which allows you to move to a more precise definition of GTE design parameters by using computational methods, summarized in Table 1. The data on the dimensions of various problems available from experiments carried out by TURBOMACHIN company. Number of processor cores is determined by a rule of thumb, proven-based on TURBOMACHIN practical experience, according to which the optimal price/performance ratio balance of the system is achieved when a one single core for every $0.2 \mathrm{~Gb}$ of memory is assigned. Upon that all problems are steady ( $t=$ const) and unsteady ( $t \neq$ const) formulated. It should also be noted that, in each case, the computation of the single sector of engine wheelspace is considered. For computation of the entire $\left(360^{\circ}\right.$ sector) wheelspace, an adequate number of times increasing of required resources is needed. For example, for complete unsteady calculation of combustion chamber $2.5 \mathrm{~Tb}$ of RAM and a 12,350 processor cores are required, that at today's level of development of microprocessor technology, roughly equivalent to the system capacity of 150 teraflops.

Represented data indicates the potential limitations that, national GTE designers, maintaining competence up to the world standards, faced. In addition to the mentioned above challenges of the experimental studies of various processes and the development of new mathematical models based on them, the development of numerical methods and program coding of these methods that supports the efficient parallelization to 1,000-10,000 cores are is required. It is worthy of note that, at present time, the domestic software products for the computational study of aerodynamics of impeller machines, heat and mass transfer and combustion processes are not available, industry companies are forced to use foreign commercial software packages that allow effective parallelization for no more than 100 cores. At the same time, leading international airspace companies have their own proprietary software that allows solving these tasks.

\section{Multicriteria Optimization}

Multicriteria optimization allows by means of a calculation find the most effective combination of product parameters before starting the manufacturing of prototype models. Let us without loss of generality consider the multicriteria minimizing problem with $\mathrm{m}$ independent variables, $n$ objectives, $P$ inequality constraints and $q$ equality constraints [14]:

$$
\text { Minimize } f(x)
$$

$$
\text { Subject to } \quad \mathrm{g}(x) \geq 0, h(x)=0
$$

where, $x=x-\left(x_{1}, \ldots, x_{m}\right) \in X, x$-decision vector (independent variables), $X$-parameter space, $f(x)^{T}=\left[f_{1}(x), \ldots, f_{n}(x)\right] \quad$-objectives, $g(x)^{T}\left[g_{1}(x), \ldots, g_{p 1}(x)\right]$-inequality constraints, $h(x)^{T}=\left[h_{1}(x), \ldots, h_{q}(x)\right]$-equality constraints. 
Decision vector $a \in X$-dominates the vector $b \in X$ (symbolyzed by $a<b$ if $\forall i \in\{1, \ldots, n\}: f_{i}(a) \leq$ $f_{i}(b) \wedge \exists j \epsilon\{1, \ldots, n\}: f_{i}(a)<f_{i}(b)$. Vector $a$ is called nondominated $X^{\prime} \subseteq X$ if in $X^{\prime}$ there is no vector dominated $a$. set of decisions $X^{\prime}$, such that:

$\forall a^{\prime} \in X^{\prime}: \neg \exists a \in X: a<a^{\prime} \wedge\left\|a-a^{\prime}\right\|<\varepsilon \wedge$

$$
\left\|f(a)-f\left(a^{\prime}\right)\right\|<\delta
$$

where, $\varepsilon>0, \delta>0$, is called local Pareto-optimal set. $X^{\prime}$ is a global Pareto-optimal set if $\forall a^{\prime} \in$ $X^{\prime}: \neg \exists a \in X: \mathrm{a}<\mathrm{a}^{\prime}$.

Thus, the problem of multi-criteria optimization resolves itself to a determination of a global Pareto-optimal solution set. Up to the date, a number of multi-criteria optimization methods, based on the nonlinear programming, genetic algorithms, etc., that used, among others, in the design of gas turbine engines, is known. One of the most efficient algorithms for multi-criteria constrained optimization is genetic algorithm Non-dominated Sorting Genetic Algorithm NSGA (non-dominated sorting genetic algorithm)-II [15]. The exception of this algorithm is that, at each step of the calculation, a new population of $N$ solutions generated, each of them the functions $f(x), g(x)$, $g(h)$. must be calculated. Population of 100 decisions, which is evolving for 500 generations, is a typical feature. Easy to estimate that, in this case, 50,000 evaluations of function $f(x), g(x), g(h)$ is required. Thus, for practical reasons, in order to achieve a reasonable computation time, a method for identifying of Pareto-optimal solution set for at least 500 calculations of accurate models of the studied correlations must be provided. To accomplish this, the approaches based on the use of the approximate models instead of correlations (1), so-called response surface models (response surface model-RSM) are used. There is a wide range of methods for RSM design-from the simplest, based on the least squares method to the more sophisticated, such as the group method of data handling, radial basis function network, and others. Generally, such models are design based on the training set, which created using one of the methods of experimental design (design of experiment-DOE). Use of these techniques allows reducing the required amount of evaluations of function $f(x), g(x)$ and $g(h)$ by two orders of magnitude [16].

Based on the data shown in Table 1, the dimensionality of the multi-criteria optimization problem should be assessed.

For example, for the engine SaM146, jointly designed by TURBOMACHINE and Snecma (France) and having a single stage fan, three stage low-pressure compressor, 6 stage high pressure compressor and, respectively, 1 and 3 stage high and low pressure turbines, the single evaluations of the function $f(x)$, $g(x)$ and $g(h)$ (calculation of the sector using the unsteady formulation) can be performed by a system with $0.65 \mathrm{~Tb}$ of RAM and 2,800 processors in $40 \mathrm{~h}$. This estimate obtained by counting the total number of floating-point operations to be performed to calculate all the elements of introduced design and evaluation of performance of mentioned system (about 33 teraflops). It is possible to estimate that, with such formulation, using RSM (500 calculations of correlations (1)), the complete optimization of the product design would take 2.3 years. Complete unsteady calculation of such engine of $360^{\circ}$ sector (with the appropriate software) would take more than $60 \mathrm{~h}$ by a system that has $11 \mathrm{~Tb}$ of RAM and 53,750 processors (630 teraflops). Design optimization, based on approximate models, would take more than 4 years, which is comparable to the design cycle of a new engine ( 7 years). It should be noted that, these estimates provide a lower bound of the time required, because in addition to the above studies should be the blade separation calculations, bird strike, acoustic calculations, etc. should be performed, that was not taken into consideration.

In addition, it should be noted that, the robustness of determined optimal solution (robust analysis) currently becomes the area of growing attention. In the real world, independent variables $X$ can have stochastic deviations from the values, which determined as the optimal values $f(x)$. This may be a result of 
deviations in the manufacturing process, which in its turn leads to a change of geometrical parameters or the chemical composition or product parts. That is why very important to estimate the impact of random variations $X$ in regard of their probabilistic characteristics on the final value $f(x)$, in particular, using Monte Carlo method. Accordingly, all calculations must be performed $\prod_{i=1}^{m} n_{i}$ times, where $n$-the vector, containing the number of variations of components of the vector $X$.

Therefore, it may be concluded that, even the use of petascale performance systems will not solve the problems of optimizing of the whole engine in the transient regime and investigate the robustness of determined optimal solution using models LES/DES/DNS (direct numerical simulation). It is obvious that, in the near future, the optimization problems will be solved whether for its specific elements or for the whole product, but using simplified models.

\section{Conclusions}

From the presented above follows, that the challenge of maintaining of up to the world standards competence in the design of GTE requires significant investments, in a first place, in creation of the experimental base for the study of aerodynamics of impeller machines, heat and mass transfer and combustion, and, secondly, in creation of the national software packages for engineering analysis working effectively in an environment with hundreds and thousands of cores.

Advanced gas turbines operating at extremely high temperatures, it is necessary to implement various cooling methods, so the turbine blades survive in the path of the hot gases. Simply passing coolant air through the airfoils does not provide adequate cooling; therefore, it is necessary to implement techniques that will further enhance the heat transfer from the airfoil walls. The internal heat transfer can be enhanced with jet impingement, pin-fin cooling and internal passages lined with turbulence promoters. The heat transfer distribution in cooling channels with turbulators has been studied for many years because a number of factors combine to affect the heat transfer.

\section{References}

[1] Merchant, A., Kerrebrock, J. L., Adamczyk, J. J., and Braunchidel, E. 2002. "Experimental Investigation of a High Pressure Ratio Aspirated Fan Stage." Presented at the ASME Turbo Expo 2004, Vienna, Austria.

[2] Lee, K. 2004. Basics CAD (SAL/CAM/CAE). SPb.: Peter, 560.

[3] Prahalad, C. K., and Hamel, G. 1990. "The Core Competence of the Corporation." Harvard Business Review 66 (May-June): 79-90.

[4] Shmotin, N. 2005. Numerical Simulation of Transient Phenomena in Gas Turbine Engines. Moscow: Innovation Engineering, 536.

[5] Shmotin, N. 2007. "Influence of the Mathematical Model to Predict the Reliability of the Heat Transfer Coefficient on the Surface of the Turbine Blade." Interdisciplinary Scientific and Technical Journal Conversion in Mechanical Engineering 4-5 (83-84): 48-55.

[6] Han, J. C., Dutta, S., and Ekkad, S. V. 2000. Gas Turbine Heat Transfer and Cooling Technology. New York: Taylor \& Francis, Inc.

[7] Metzger, D. E., Florschuetz, L. W., Takeuchi, D. I., Behee, R. D., and Berry, R. A. 1979. "Heat Transfer Characteristics for Inline and Staggered Arrays of Circular Jets with Crossflow of Spent Air." ASME Journal of Heat Transfer 101 (3): 526-31.

[8] Florschuetz, L. W., and Su, C. C. 1987. "Effects of Crossflow Temperature on Heat Transfer within an Array of Impinging Jets." ASME Journal of Heat Transfer 109 (1) $74-82$.

[9] Metzger, D. E., Berry, R. A., and Bronson, J. P. 1982. "Developing Heat Transfer in Rectangular Ducts with Staggered Arrays of Short Pin Fins." ASME Journal of Heat Transfer 104 (4): 700-6.

[10] Chyu, M. K., Hsing, Y. C., Shih, T. I. P., and Natarajan, V. 1998. "Heat Transfer Contributions of Pins and Endwall in Pin-Fin Arrays: Effects of Thermal Boundary Condition Modeling." ASME Paper No. 98-GT-175.

[11] Metzger, D. E., Fan, S. C., and Haley, S. W. 1984. "Effects of Pin Shape and Array Orientation on Heat Transfer and Pressure Loss in Pin Fin Arrays." ASME Journal of Engineering for Gas Turbines and Power 106 (1): 252-7.

[12] Chyu, M. K. 1990. "Heat Transfer and Pressure Drop for Short Pin-Fin Arrays with Pin-Endwall Fillet." ASME J. of Heat Transfer 112 (4): 926-32.

[13] Chyu, M. K., Hsing, Y. C., and Natarajan, V. 1998. 
"Convective Heat Transfer of Cubic Fin Arrays in a Narrow Channel." ASME Journal of Turbomachinery 120 (2): 362-7.

[14] Liu, G. P., Yang, J. B., and Whidborne, J. F. 2003. Multi-objective Optimization and Control. Baldock, England: Research Studies Press Ltd., 330.

[15] Deb, K. S., Agrawal, A. P., and Meyarivan, T. 2000. "A
Fast Elitist Non-sorting Algorithm for Multi-objective: NASA-II." In Proceedings of the Parallel Problem Solving from Nature VI, 849-58.

[16] Zelenkov, Y. A. 2010. "The Method of Multi-criteria Optimization Based on Approximate Models of the Object." Numerical Methods and Programming 11 (2): 92-102. 